\title{
Einfluss der medizinischen computerassistierten Telefontriage auf das Patientenverhalten: erste Erfahrungen in der Schweiz
}

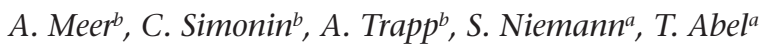

\section{Zusammenfassung}

Seit dem Frühjahr 2000 wird in der Schweiz durch die Firma Medvantis medi-24 eine medizinische computerassistierte Telefontriage angeboten. Die wissenschaftliche Evaluation der neuen Dienstleistung ist Teil des Qualitätssicherungsprozesses. Aus diesem Grund hat Medvantis medi-24 das Institut für Sozial- und Präventivmedizin der Universität Bern (ISPM) mit der Durchführung der vorliegenden Studie beauftragt.

$70 \%$ der Anrufenden wichen in der Selbstbeurteilung ihrer Beschwerden hinsichtlich der notwendigen Handlung und deren Dringlichkeit von der Empfehlung der Fachpersonen des Medizinischen Expertenzentrums ab. $63 \%$ der Befragten änderten, nach der Beratung, ihre ursprüngliche Handlungsabsicht. Über $90 \%$ der schriftlich befragten nachbehandelnden Ärzte beurteilten die durch das Medizinische Expertenzentrum veranlassten Konsultationen und deren Zeitpunkt als gerechtfertigt.

Die Studie ergibt Hinweise, dass medizinische Laien in der Selbstbeurteilung der Dignität ihrer Gesundheitsbeschwerden oft überfordert sind und in diesem Bereich ein Informationsdefizit vorliegt. Die relativ hohe Compliance bezüglich des Ratschlages des Medizinischen Expertenzentrums weist auf die Effektivität der Dienstleistung hin und zeigt, dass das neue Instrument im schweizerischen Gesundheitswesen einen Einfluss auf das Patientenverhalten hat. Die Methodik zur Evaluation der Qualität der Triageempfehlung muss weiterentwickelt werden. Eine gesundheitsökonomische Relevanz im Sinne einer Kostenminderung wird vermutet.

\section{Einleitung}

In der Schweiz bietet die Firma Medvantis medi24 seit dem Frühjahr 2000 die Dienstleistungen eines Medizinischen Expertenzentrums an. Patienten sollen einerseits sachlich und unabhängig zu medizinischen Fragen informiert und anderseits bei Beschwerden bezüglich des optimalen Behandlungspfades beraten werden. Die Gesundheitsberaterinnen werden durch ein computerbasiertes medizinisches Expertensystem in der symptomorientierten und strukturierten Befragung sowie in der Triage unterstützt. Aufgrund des Fachwissens der Gesundheitsberaterinnen und der Ärzte sowie der Entscheidungsunterstützung durch das Computersystem wird den Anrufenden eine der in Tabelle 2 aufgeführten grundsätzlichen Handlungsempfehlungen gegeben.

Bestehende und neue Dienstleistungen im Gesundheitswesen haben ihr Angebot auf Effizienz, Effektivität und Qualität zu überprüfen. Aus diesem Grund hat Medvantis Schweiz zusätzlich zu den internen Qualitätskontrollen das Institut für Sozial- und Präventivmedizin der Universität Bern (ISPM) beauftragt, die Dienstleistung in einem permanenten Prozess wissenschaftlichen $\mathrm{zu}$ evaluieren. Die vorliegende Studie fasst die ersten Resultate zusammen.

\section{Tabelle 1}

Wichtigste Erkenntnisse.

$70 \%$ der Anrufenden beurteilten ihre Beschwerden hinsichtlich der notwendigen Handlung und deren Dringlichkeit anders, als dies Fachleute des Medizinischen Expertenzentrums taten.

- $63 \%$ der Befragten änderten nach der Beratung durch die Gesundheitsberaterin ihre ursprüngliche Handlungsabsicht.

- Nur $2 \%$ aller Patienten, die vorhatten, eine Notfallstation aufzusuchen, wurde dieses Vorhaben bestätigt.

- $81 \%$ der Anrufenden mit der Absicht, direkt eine Notfallstation aufzusuchen, haben sich nach der Beratung selbst oder von ihrem Hausarzt oder dessen Vertretung behandeln können.

- $90 \%$ der schriftlich befragten nachbehandelnden Ärzte beurteilten die nach der Beratung durch das Medizinische Expertenzentrum erfolgten Konsultationen und deren Zeitpunkt als gerechtfertigt.

- Medizinische Laien sind in der Selbstbeurteilung der Dignität ihrer Gesundheitsbeschwerden oft überfordert. 
Tabelle 2

Triagestufen, Bezeichnungen und Handlungsempfehlungen.

\begin{tabular}{lll}
$\begin{array}{l}\text { Triagestufe } \\
1\end{array}$ & Bezeichnung & Handlungsempfehlung \\
\hline 2 & Arztbesuch sofort (AZ/S) & $\begin{array}{l}\text { Vermittlung an den regionalen Rettungs- } \\
\text { dienst 144 mit der Empfehlung der notfall- } \\
\text { mässigen stationären Weiterbetreuung. }\end{array}$ \\
\hline 3 & $\begin{array}{l}\text { Empfehlung zur sofortigen ambulanten } \\
\text { Notfallkonsultation durch den Hausarzt oder } \\
\text { dessen Vertretung. }\end{array}$ \\
\hline 4 & Arztbesuch $<24$ Stunden (AZ/R) & $\begin{array}{l}\text { Empfehlung zur Konsultation beim Haus- } \\
\text { arzt oder bei dessen Vertretung innerhalb } \\
24 \text { Stunden. }\end{array}$ \\
\hline 5 & Arztbesuch Routine (AZ/R) & $\begin{array}{l}\text { Empfehlung zur Hausarztkonsultation inner- } \\
\text { halb 2-7 Tagen. }\end{array}$ \\
\hline Selbstbehandlung (SB) & $\begin{array}{l}\text { Keine Arztkonsultation. Die Gesundheits- } \\
\text { beraterin erteilt Ratschläge zur Selbstbehand- } \\
\text { lung. }\end{array}$
\end{tabular}

\section{Material und Methoden}

Vom 26. Februar bis zum 30. Juni 2001 sowie vom 15. Oktober 2001 bis zum 5. Februar 2002 wurden über 2000 Anrufende am Anfang des Beratungsgesprächs nach ihrer Handlungsabsicht und nach der Selbsteinschätzung in bezug auf die Behandlungsdringlichkeit ihrer Beschwerden gefragt. Die Absicht wurde dann mit der Beurteilung der Gesundheitsberaterin am Ende der computerassistierten Telefontriage verglichen. Vor Beendigung des Gespräches wurde von den Anrufenden das mündliche Einverständnis zu einer Nachbefragung durch das ISPM eingeholt. 7-10 Tage nach dem Kontakt mit dem Medizinischen Expertenzentrum wurde die Studienpopulation durch Mitarbeitende des ISPM telefonisch nach der tatsächlich erfolgten Handlung und deren zeitlichem Abstand zum Beratungsgespräch befragt. Sämtliche Studienfragen waren offen formuliert, wobei die Antworten einer von 13 möglichen Handlungsabsichten und 6 Dringlichkeitsstufen zugeordnet wurden. Mit der offenen Fragestellung wurde beabsichtigt, den Bias durch die Befragung selbst möglichst klein zu halten. Die 78 verschiedenen Antwortkombinationen wurden über eine Matrix den 5 verschiedenen Triagestufen des Medizinischen Expertenzentrums zugeordnet. Zusätzlich wurde eine sechste Triagegruppe definiert («Triagegruppe 6»), welche diejenigen Anrufenden beinhaltet, die explizit angaben, ihre Handlungsabsicht auf eine fachkundige, medizinische Telefonberatung abzustützen. Die Matrix wurde vor der Datenerhebung aufgrund einer Konsensuskonferenz von medizinischen und paramedizinischen Vertretern des ISPM und des Medizinischen Expertenzentrums festgelegt.
Die drei Erhebungen: Handlungsabsicht/ Dringlichkeit, Triageergebnis/Dringlichkeit und die tatsächlich erfolgte Handlung bzw. deren zeitliche Latenz zum Kontakt mit dem Expertenzentrum wurden miteinander verglichen und hinsichtlich der folgenden beiden Evaluationskriterien untersucht:

1. Abweichung der Handlungsabsicht/Dringlichkeit von der Handlungsempfehlung des Medizinischen Expertenzentrums;

2. Befolgung des vom Medizinischen Expertenzentrum erteilten Ratschlages (Compliance).

Die Studiendaten wurden deskriptiv- und inferenz-statistisch ausgewertet. In dieser Publikation werden die Daten vorwiegend deskriptiv-statistisch dargestellt. Die Signifikanzwerte erscheinen in der Diskussion im Kontext und beruhen auf dem McNemar-Testverfahren. Die McNemar- $\mathrm{X}^{2}$-Statistik berechnet sich durch: $\mathrm{X}^{2}=(\mathrm{b}-\mathrm{c})^{2} / \mathrm{b}+\mathrm{c}(\mathrm{df}=1)$.

Die zweiwertigen Daten (Freiheitsgrad df $=1$ ) der Absichten und der Triageempfehlungen bzw. der Absichten und des Verhaltens lassen sich in Vierfeldertafeln (Felder a-d) darstellen. Der McNemar-Test vergleicht die Anteile der Auf- oder Rückstufungen der Felder b und c (Off-Diagonale) und testet deren Proportion auf Signifikanz. Geprüft wird die Nullhypothese, dass die Proportionen des Wechsels von b zu c $\left(\mathrm{p}^{\prime} \mathrm{b}\right)$ und umgekehrt $\left(\mathrm{p}_{\mathrm{C}}^{\prime}\right)$ gleich sind: $\mathrm{p}^{\prime}=\mathrm{p}_{\mathrm{b}}^{\prime}=\mathrm{p}_{\mathrm{C}}^{\prime}=\left(\mathrm{p}_{\mathrm{b}}^{\prime}+\mathrm{p}_{\mathrm{C}}^{\prime}\right) / 2$.

Medvantis Schweiz beschäftigt 4 Fachärzte sowie 18 medizinische Fachkräfte mit einer fundierten Grundausbildung in einem Pflegeberuf und einer für die Beratungstätigkeit in einem Medizinischen Expertenzentrum konzipierten, betriebsinternen Zusatzausbildung. Die durchschnittliche praktische Berufserfahrung der Gesundheitsberaterinnen beträgt 10 Jahre. Der Beratungsprozess und dessen Dokumentation wird durch ein Informationssystem elektronisch abgebildet (TAS/Plain Software Company/GB).

In der zweiten Datenerhebungsphase wurde eine schriftliche Befragung der nachbehandelnden Ärzte durchgeführt. Während des Interviews durch das ISPM sind die Anrufenden, welche zwischenzeitlich einen Arzt aufgesucht hatten, gefragt worden, ob sie damit einverstanden sind, dass auch der behandelnde Arzt in die Studie miteinbezogen wird. Dieser nahm Stellung dazu, ob die nach dem Kontakt mit dem Expertenzentrum erfolgte Konsultation gerechtfertigt und der Zeitpunkt der Konsultation korrekt gewählt wurde. Die Triageempfehlung wurde den beurteilenden Ärzten seitens des ISPM nicht mitgeteilt. 
Abbildung 1

Häufigkeitsverteilung Absicht $(n=1614)$.

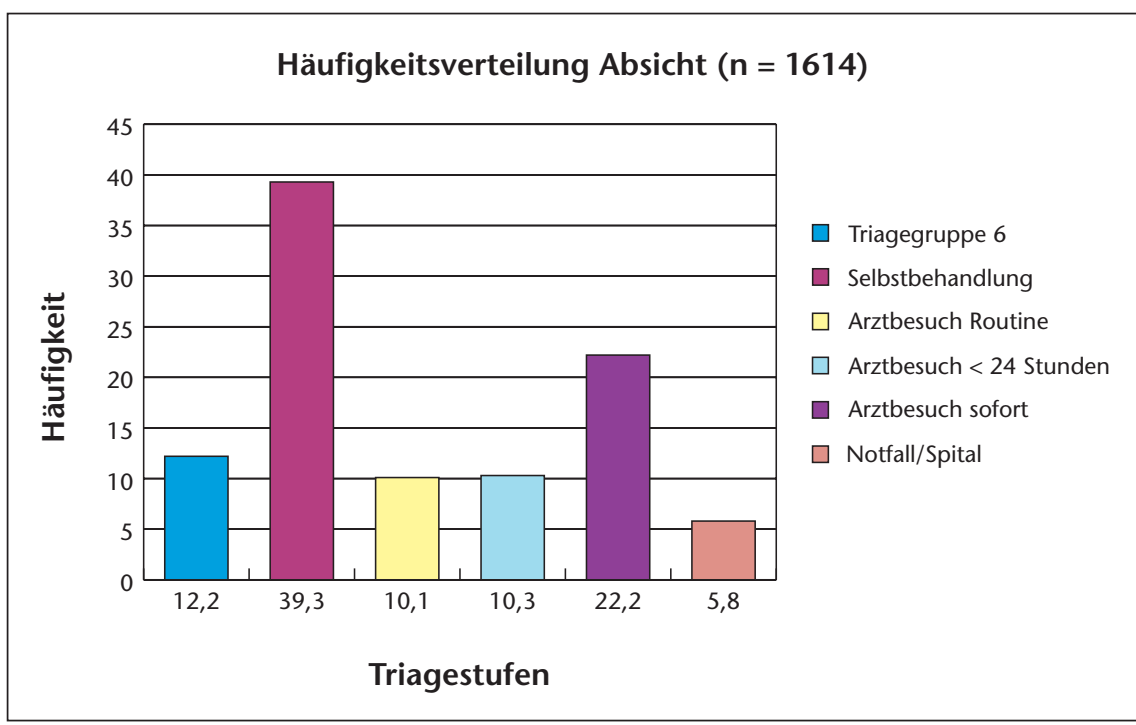

Abbildung 2

Häufigkeitsverteilung Triage $(n=1614)$.

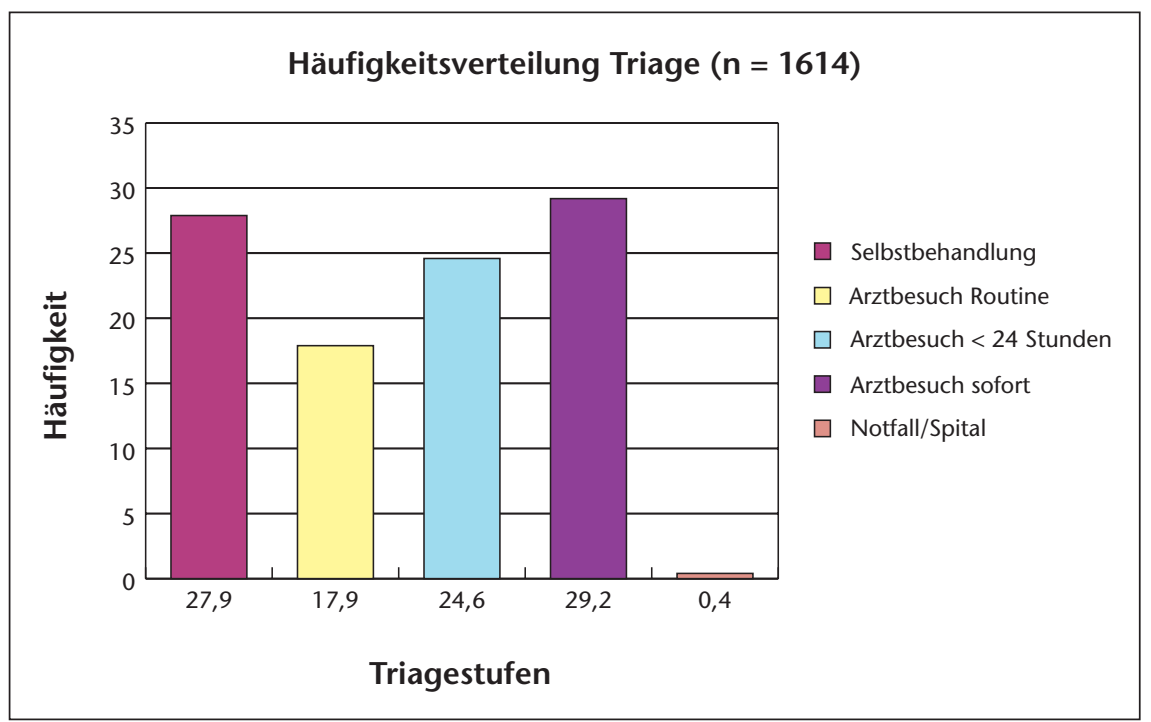

\section{Resultate}

Von den 2092 Anrufenden, welche ihr Einverständnis zum Rückruf gegeben hatten, konnten durch das ISPM 1743 Haushalte kontaktiert werden. 129 Erhebungen konnten keiner der 78 Antwortkombinationen zugeordnet werden und wurden deshalb aus der Studie ausgeschlossen. Von den 1614 ausgewerteten Anrufen äusserten 197 (12\%) der Befragten keine klare Handlungsabsicht und gaben an, dass sie sich telefonisch bei einer medizinisch geschulten Person beraten lassen hätten. Sie wurden der «Triagegruppe 6»
(TG6) zugeordnet. Die prozentualen Häufigkeitsverteilungen der verschiedenen Triagestufen sind in den Abbildungen 1-3 dargestellt.

Die Gegenüberstellungen Absicht versus Triage, Triage versus Verhalten und Absicht versus Verhalten sind in den Abbildungen 4-6 zusammengestellt. Dabei wurden die Triagestufen «Arztbesuch <24 Std.» und «Arztbesuch Routine» $\mathrm{zu}$ «Arztbesuch regulär» (AZ/R) aggregiert. Die Daten werden dadurch einer gesundheitsökonomischen Sichtweise besser gerecht, da hier die Unterscheidungen ambulante oder stationäre notfallmässige Konsultation, regulärer Arztbesuch oder Selbstbehandlung bedeutsam sind.

Insgesamt $70 \%$ der Anrufenden wichen in der Selbstbeurteilung ihrer Beschwerden hinsichtlich der notwendigen Handlung und deren Dringlichkeit von der Beurteilung durch die medizinischen Fachperson ab. $63 \%$ der Befragten änderten nach dem telefonischen Kontakt mit dem Medizinischen Expertenzentrum ihre ursprüngliche Handlungsabsicht. Die mittlere Compliance aller Triagestufen betrug $53 \%$.

Der Vergleich der Handlungsabsicht vor der Beratung durch das Medizinische Expertenzentrum mit dem Verhalten danach ergibt insgesamt 426 Rückstufungen zu kostengünstigeren und 405 Aufstufungen zu kostenintensiveren Versorgungsstufen. Die relativen Häufigkeiten der Verschiebungen zwischen den einzelnen Versorgungsstufen sind in Abbildung 7 dargestellt. Die Rückstufungen überwiegen in den kostenintensiven Notfallversorgungsbereichen (1-2: Notfall Spital zu Arztbesuch sofort, 1-3: Notfall/Spital zu Arztbesuch <24 Stunden, 1-4: Notfall/Spital zu Arztbesuch Routine, 1-5: Notfall/Spital zu Selbstbehandlung, 2-3: Arztbesuch sofort zu Arztbesuch <24 Stunden, 2-5: Arztbesuch Routine zu Selbstbehandlung). Hingegen erfolgen mehr Aufstufungen zu Routinearztkonsultationen innerhalb 24 Stunden (3-4: Arztbesuch Routine zu Arztbesuch <24 Stunden, 3-5: Selbstbehandlung zu Arztbesuch $<24$ Stunden).

291 Anrufende gaben ihr Einverständnis, dass der nachbehandelnde Arzt bezüglich der erfolgten Konsultation befragt werden durfte. 261 Ärzte wurden schriftlich erreicht und 205 Fragebogen konnten ausgewertet werden (Rücklauf $79 \%$ ). In $90 \%$ (185) der Fälle wurde die erfolgte Konsultation als gerechtfertigt erachtet, in $3 \%$ (5) wurde die Frage nicht beantwortet, während $7 \%$ (15) der Konsultationen als nicht gerechtfertigt eingestuft wurden. Der Zeitpunkt der als gerechtfertigt beurteilten Konsultationen wurde in $91 \%$ (153) als korrekt eingestuft, in $5 \%$ (10) als zu früh und in $4 \%$ (7) als zu spät beurteilt. 
Abbildung 3

Häufigkeitsverteilung Verhalten $(n=1614)$.

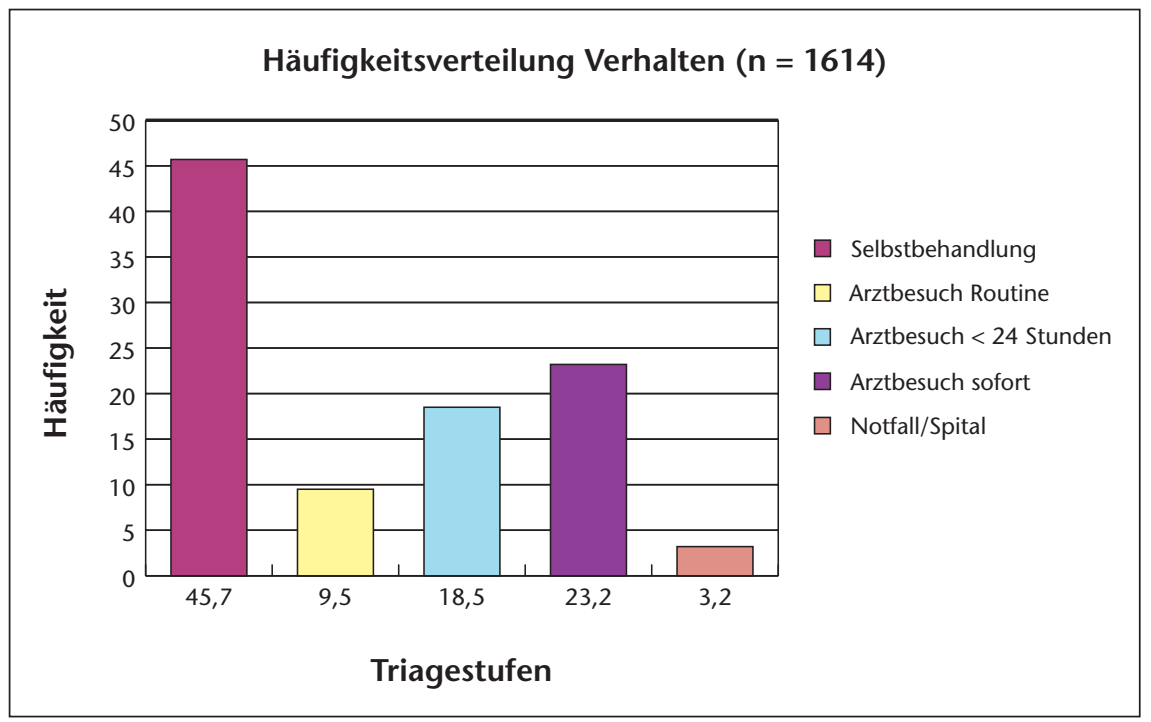

\section{Abbildung 4}

Abweichung der Triage von der Absicht der Anrufenden. Absolutzahlen der Handlungsabsichten in Klammern: NF/SP = Notfall/Spital (93), AZ/S = Arztbesuch sofort (359), AZ/R = Arzt regulär (330), SB = Selbstbehandlung (635), TG6 = Triagegruppe 6 (197).

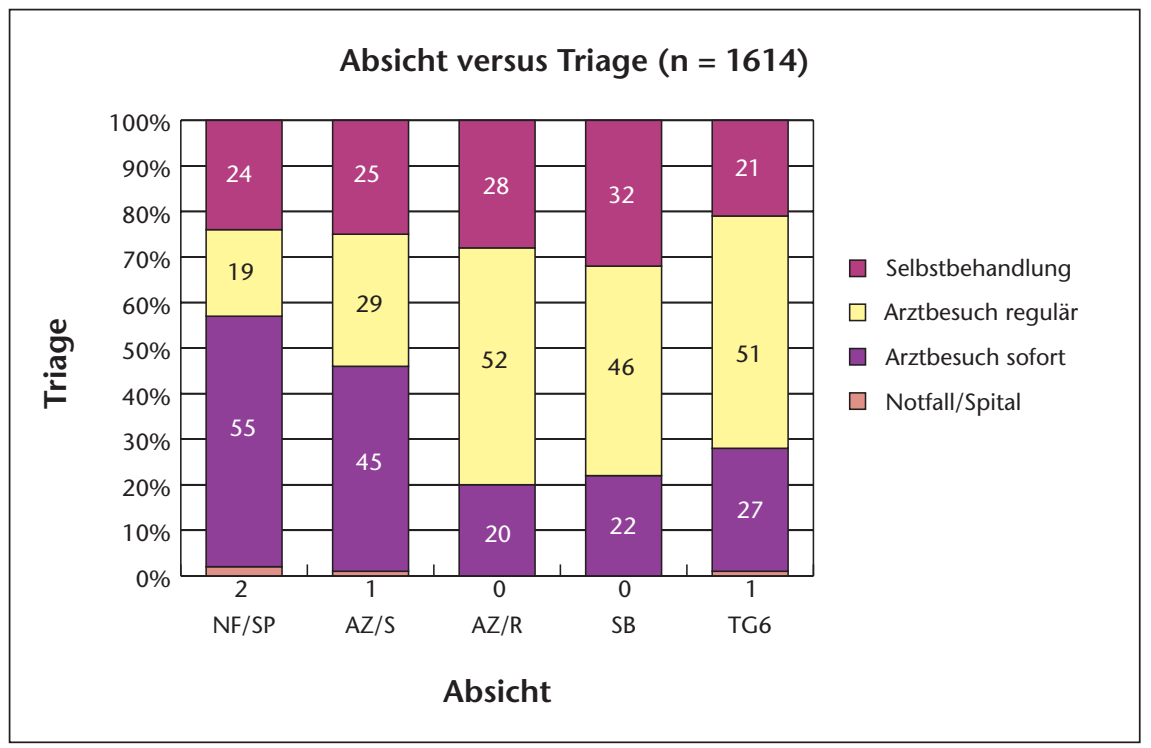

1 Munro J, Nicholl J, O’Cathain A, Knowles E. Evaluation of NHS Direct first wave sites: First interim report to the Department of Health. Sheffield: Medical Care Research Unit; 1998.
Im weiteren wird die Dienstleistung dem Kundenkreis von unterschiedlichen Institutionen (Versicherungen, Ärztegruppen, staatlichen Organen) mit verschiedenartigen Hintergründen und Zielsetzungen zur Verfügung gestellt. Aufgrund dieser Heterogenität sind der internationale Vergleich und die Übertragung von wissenschaftlichen Auswertungen und Erfahrungen im Zusammenhang mit der Dienstleistung Medizinischer Expertenzentren nur eingeschränkt möglich. Zudem ist die wissenschaftliche Evaluation Teil des Qualitätssicherungsprozesses der Dienstleistung und sollte deshalb länder- und situationsspezifisch durchgeführt werden.

Die vorliegende Studie verglich die Absicht des Anrufenden, die Handlungsempfehlung der Gesundheitsberaterin und das Verhalten des Anrufenden miteinander.

In $70 \%$ beurteilten die Anrufenden ihre Beschwerden bezüglich der notwendigen Handlung und deren Dringlichkeit anders als die Fachpersonen des Medizinischen Expertenzentrums. Eine ähnliche Untersuchung wurde von Munro et al. im Rahmen der wissenschaftlichen Evaluation von NHS Direct in England durchgeführt. In dieser Studie wichen $52 \%$ der befragten Anrufenden in ihrer Handlungsabsicht vom Triageentscheid $a b$ [1]. In der englischen Studie erfolgte die Befragung bezüglich der Handlungsabsicht eine bis mehrere Wochen nach dem Kontakt mit dem Medizinischen Expertenzentrum mittels eines schriftlichen Fragebogens. Um eine Verfälschung der Befragung nach der Handlungsabsicht durch den erteilten Ratschlag zu verhindern, erfolgte in unserer Studie die Befragung telefonisch am Anfang des Beratungsgespräches. Die vermeintlich bessere Selbstbeurteilung der englischen Studienpopulation lässt einen systematischen Fehler durch die retrospektive Befragungsmethode vermuten. Die Abweichungen waren bei den Triagestufen «Notfall/Spital sofort» $(98 \%, \mathrm{p}<0,001)$, «Arztbesuch sofort» (55\%, p <0,001) und «Selbstbehandlung» (68\%, $\mathrm{p}<0,001)$ besonders gross.

Diese Studiendaten weisen darauf hin, dass medizinische Laien in der Selbstbeurteilung der Dignität von Gesundheitsbeschwerden oft überfordert sind. Insbesondere scheint es Mühe zu bereiten, Notfallsymptome korrekt einzuschätzen und aufgrund einer bestehenden Symptomatik richtig zu entscheiden, ob eine Beschwerde selbständig behandelt werden kann oder nicht. Die Dienstleistungen eines Medizinischen Expertenzentrums erscheinen vor diesem Hintergrund sinnvoll.

Gesamthaft änderten $63 \%$ der Befragten nach dem telefonischen Kontakt mit dem Medi- 
Abbildung 5

Abweichung des Verhaltens der Anrufenden von der Absicht. Absolutzahlen der Handlungsabsichten in Klammern: NF/SP = Notfall/Spital (93), AZ/S = Arztbesuch sofort (359), AZ/R = Arzt regulär (330), SB = Selbstbehandlung (635), TG6 = Triagegruppe 6 (197).

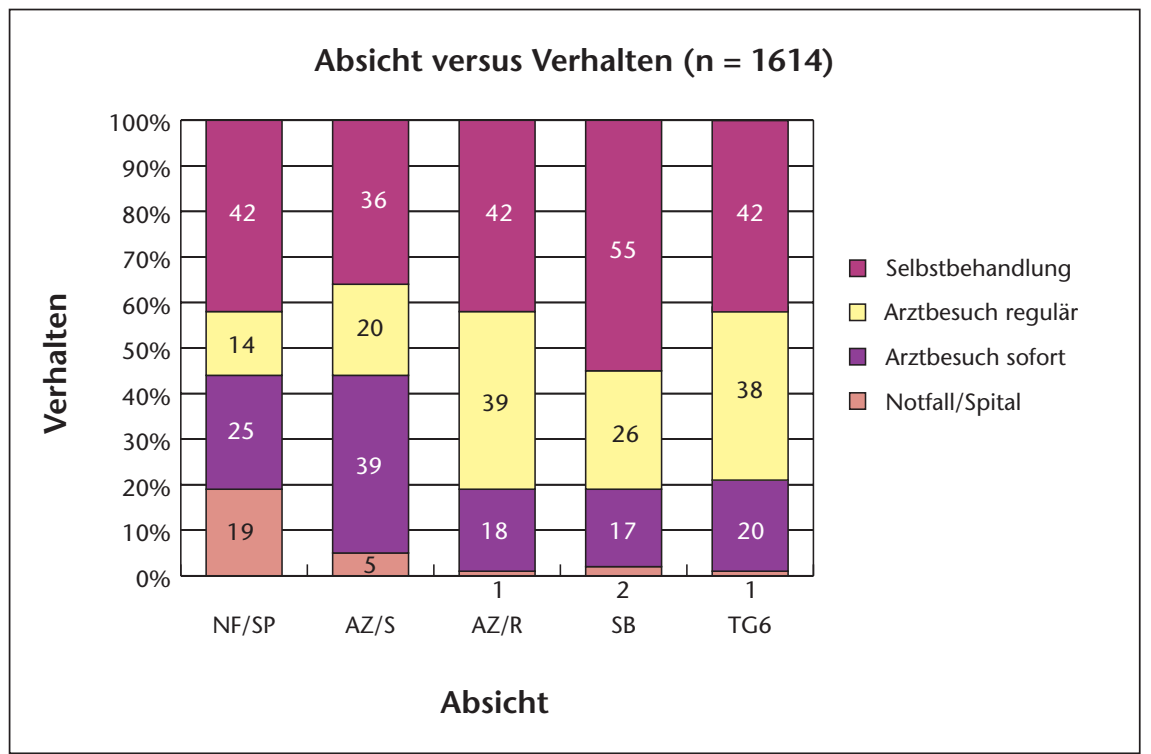

\section{Abbildung 6}

Abweichung des Verhaltens der Anrufenden vom Triageentscheid. Absolutzahlen der Triagen in Klammern: NF/SP = Notfall/Spital (6), AZ/S = Arztbesuch sofort (472), AZ/R = Arztbesuch regulär (686), SB = Selbstbehandlung (450).

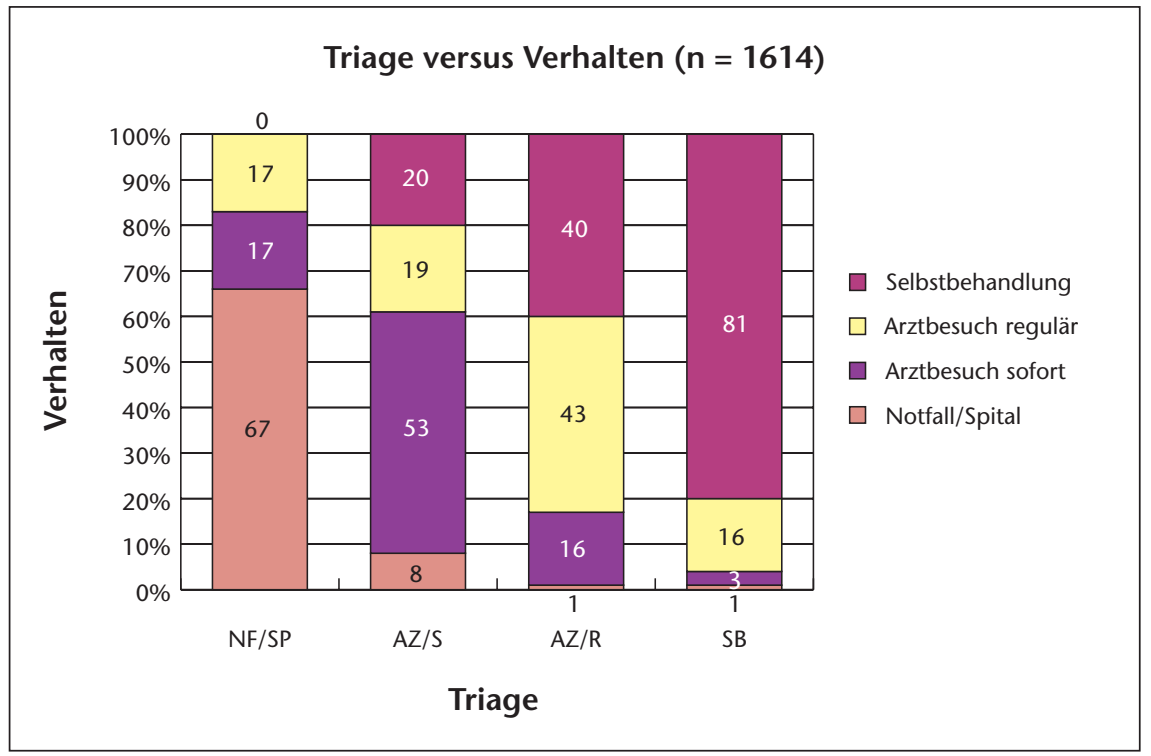

2 Flaig $\mathrm{C}$, Zehnder $\mathrm{K}$, Zürcher $\mathrm{H}$ Eichenberger P, Frei C, Gegeckas A et al. Selbsteinweisungen ins Spital. PrimaryCare 2002;2:280-3. zinischen Expertenzentrum ihre ursprüngliche Handlungsabsicht. Die Compliance war im Notfall- und Selbstbehandlungsbereich besonders hoch. Gemäss der Studie von Flaig et al. [2] an zwei Schweizer Spitälern (Kantonsspital Baden, Bezirksspital Brugg) hätten $80 \%$ der Patienten, welche sich mit Beschwerden direkt an der Notfallpforte des Spitals gemeldet haben, grundsätzlich in den Praxen der Grundversorger behandelt werden können. Die subjektive Dringlichkeit, die Abwesenheit des Hausarztes, die räumliche Nähe des Spitals und die Unkenntnis über den Notfalldienst wurden als wichtigste Gründe genannt, das Spital direkt aufzusuchen, ohne vorher den Haus- oder Notfallarzt zu kontaktieren.

Die vorliegenden Daten bestätigen die obgenannte Beobachtung einer inadäquaten medizinischen Ressourcennutzung seitens der Patienten. Lediglich 2\% der Anrufenden wurden von der Gesundheitsberaterin in ihrer Absicht bestärkt, direkt die Notfallstation des Spitals aufzusuchen. $81 \%(\mathrm{p}<0,001)$ der Anrufenden mit dieser Absicht sind nach der Beratung von ihrem ursprünglichen Vorhaben abgewichen und haben sich selbst behandeln können oder vom Hausarzt oder dessen Vertretung behandeln lassen. Die Beratung durch ein medizinisches Expertenzentrum erscheint effektiv und ermöglicht eine Prozessoptimierung und verbesserte Ressourcenallokation. Die Dienstleistung eines Medizinischen Expertenzentrums kann einer medizinischen Über- und Unterversorgung der Schweizer Bevölkerung entgegenwirken.

In der zweiten Datenerhebungsphase wurde zusätzlich eine schriftliche Befragung der nachbehandelnden Ärzte durchgeführt. Über $90 \%$ der befragten Ärzte beurteilten die Konsultation und den Zeitpunkt der Konsultation als gerechtfertigt. Es handelt sich dabei um eine preliminäre Datenerhebung mit der Absicht, eine weitere Methodik zur Evaluation der Dienstleistung aufgrund der Beurteilung der nachbehandelnden Ärzte zu erarbeiten. Die Informationen sind rein deskriptiver Natur und können nicht direkt als Instrument zur Qualitätsbeurteilung der Triageempfehlungen verwendet werden.

Die Qualitätsbeurteilung medizinischer Dienstleistungen ist grundsätzlich schwierig. Auch die Beurteilung der Korrektheit einer medizinischen Triage ist mehrschichtig. Im Rahmen der internen Qualitätssicherungsprozesse lässt Medvantis medi-24 die aufgezeichneten Triagegespräche und die sich daraus ergebenden Empfehlungen durch verschiedene Experteninstanzen (unabhängige Grundversorger, Ärzteschaft des Medizinischen Expertenzentrums, nichtärztliche medizinische Fachpersonen) 


\section{Abbildung 7}

Die Rückstufungen überwiegen in den kostenintensiven Notfallversorgungsbereichen (1-2: Notfall Spital zu Arztbesuch sofort, 1-3: Notfall/Spital zu Arztbesuch <24 Stunden, 1-4: Notfall/Spital zu Arztbesuch Routine, 1-5: Notfall/Spital zu Selbstbehandlung, 2-3: Arztbesuch sofort zu Arztbesuch $<24$ Stunden, 2-5: Arztbesuch Routine zu Selbstbehandlung). Hingegen erfolgen mehr Aufstufungen zu Routinearztkonsultationen innerhalb 24 Stunden (3-4: Arztbesuch Routine zu Arztbesuch $<24$ Stunden, 3-5: Selbstbehandlung zu Arztbesuch <24 Stunden).

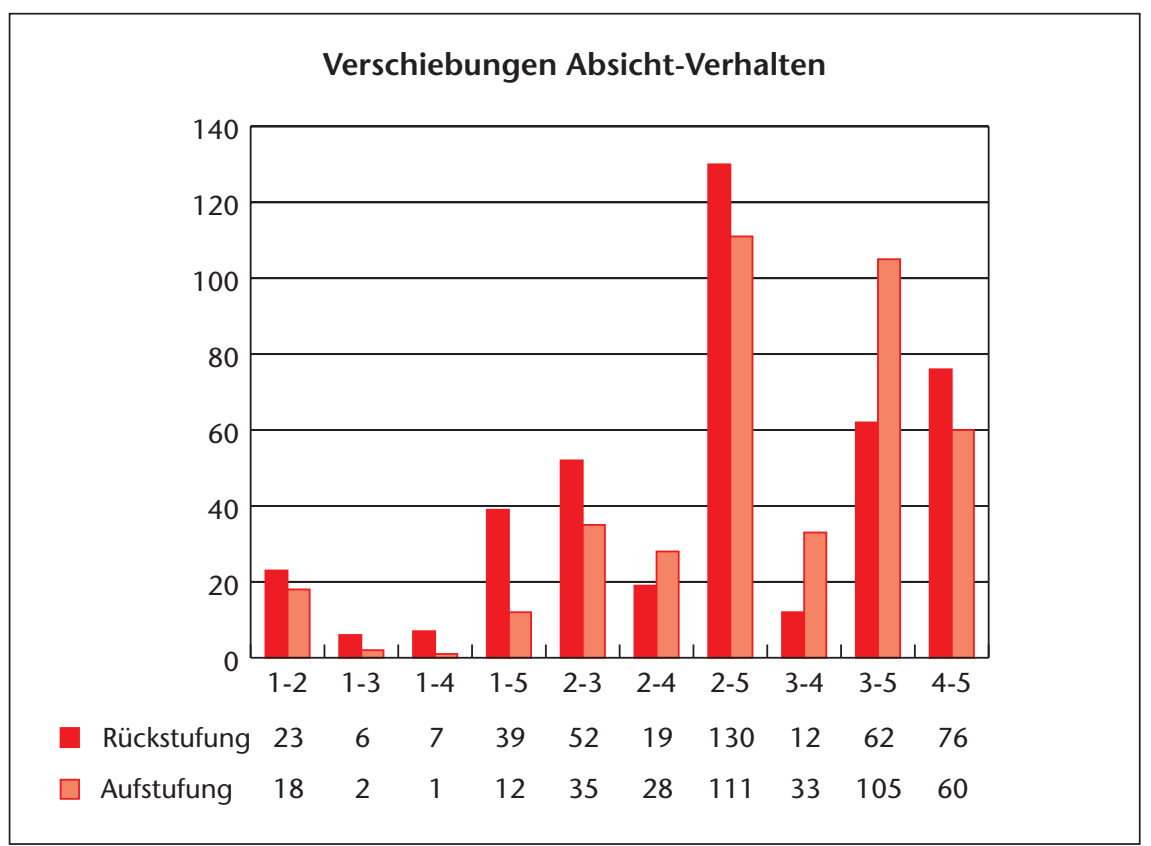

3 Munro J, Nicholl J, O'Cathain A, Knowles E, Morgan A. Evaluation of NHD Direct first wave sites: Final report of the phase 1 research. Sheffield: Medical Care Research Unit; 2001.

4 Lattimer V, Sassi F, Gorge S, Moore M, Turnbull J, Mullee M et al. Cost analysis of nurse telephone consultation in out of hours primary care: evidence from a randomised controlled trial. Br Med J 2000;320:1053-7. evaluieren. Im Schlussbericht der ersten Untersuchungsphase von NHS Direct [3] wird auf die lung durch die ärztlichen Experten hingewiesen und eine erweiterte Qualitätssicherungsmethodik angeregt. Die Qualitätsbeurteilung sollte sich auf ein Expertenpanel mit einem möglichst breiten medizinischen Hintergrund stützen und die Triageratschläge sowohl im Zusammenhang mit den zum Zeitpunkt der Befragung erhobenen als auch den zu diesem Zeitpunkt verfügbaren Informationen beurteilt werden. Zusätzlich wird angeregt, die Triagedienstleistung in Kohortenstudien ähnlich einem Screeningtest auf ihre Sensitivität (z. B. Anteil der Patienten, welche triagiert wurden) und ihre Spezifität (z. B. Anteile der Patienten, welchen korrekterweise keine Notfallbehandlung empfohlen wurde) zu untersuchen. Die Erarbeitung einer erweiterten Methodik zur Qualitätssicherung ist Gegenstand der fortgeführten wissenschaftlichen Evaluation der Dienstleistungen von Medvantis medi-24 durch das ISPM.

Die untersuchte Triagedienstleistung wird auch als Demand Management bezeichnet. Demand Management ist eine Form der Patientenführung mit dem Ziel, einen optimalen Bezug hohe interindividuelle Variabilität der Beurteieiner Notfallkonsultation bedürfen und korrekt von medizinischen Leistungen sicherzustellen. Die Förderung einer kostengünstigen Medizin ist ein zentrales, aber nicht primäres Anliegen dieses Ansatzes. Seit der Einführung Medizinischer Expertenzentren wurde deren gesundheitsökonomischen Relevanz besondere Bedeutung zugemessen. Das Design dieser Studie ist nicht darauf ausgerichtet, gesundheitsökonomische Aussagen zu machen. Die erhobenen Daten sollen deshalb nur kurz in diesem Zusammenhang diskutiert werden.

Aus gesundheitsökonomischer Sicht können direkte und indirekte Kostenfolgen unterschieden werden. Die verschiedenen Triagebereiche sind in bezug auf ihre direkten Folgekosten nicht homogen. Eine unnötige Notfallkonsultation an einem Spital verursacht ungleich mehr Kosten als eine ungerechtfertigte reguläre ambulante Arztkonsultation.

Insgesamt ergibt der Vergleich der Handlungsabsicht vor der Beratung durch das Medizinische Expertenzentrum mit dem Verhalten danach eine Nettoverschiebung zu kostengünstigeren Versorgungsstufen. Die Verschiebung zeigt sich vor allem in den kostenintensiven Notfallbereichen (Notfall Spital, HA/NA sofort). Lattimer et al. [4] wiesen in einer randomisierten Studie die Kosteneffizienz der Telefontriagedienstleistung für den Notfalldienst einer Grundversorgerkooperative (55 Grundversorger/ Einzugsgebiet von 97000 Einwohnern) in Wiltshire/UK nach. Die Kosteneinsparungen erfolgten primär aufgrund einer besseren Ressourcenallokation im Notfallbereich. Zusätzlich zur direkten Kostenminderung vermuten wir eine weit höhere, indirekte Kostenreduktion aufgrund der korrekten und rechtzeitigen Zuweisung der Anrufenden zum grundversorgenden Arzt. Die Untersuchung der gesundheitsökonomischen Auswirkungen der Dienstleistungen von Medvantis medi-24 ist Gegenstand weiterer Forschungsbemühungen.

\section{Dank}

Herzlichen Dank an Frau Anne Sybil Götschi für das sorgfältige Gegenlesen und die wertvollen Anregungen. 